\title{
Dispersion of Endoplasmic Reticulum-associated Compartments by 4-phenyl Butyric Acid in Yeast Cells
}

\author{
Thanh Chi Mai ${ }^{\dagger}$, Yuki Ishiwata-Kimata ${ }^{\dagger}$, Quynh Giang Le, Hiroyuki Kido, and Yukio Kimata* \\ Division of Biological Science, Graduate School of Science and Technology, Nara Institute of Science and \\ Technology, 8916-5 Takayama, Ikoma, Nara 630-0192, Japan
}

\begin{abstract}
In yeast Saccharomyces cerevisiae cells, some aberrant multimembrane-spanning proteins are not transported to the cell surface but form and are accumulated in endoplasmic reticulum (ER)-derived subcompartments, known as the ER-associated compartments (ERACs), which are observed as puncta under fluorescence microscopy. Here we show that a mutant of the cell surface protein Pma1, Pma1-2308, was accumulated in the ERACs, as well as the heterologously expressed mammalian cystic fibrosis transmembrane conductance regulator (CFTR), in yeast cells. Pma1-2308 and CFTR were located on the same ERACs. We also note that treatment of cells with 4-phenyl butyric acid (4-PBA) compromised the ERAC formation by Pma1-2308 and CFTR, suggesting that 4-PBA exerts a chaperone-like function in yeast cells. Intriguingly, unlike ER stress induced by the canonical ER stressor tunicamycin, ER stress that was induced by Pma1-2308 was aggravated by 4-PBA. We assume that this observation demonstrates a beneficial aspect of ERACs, and thus propose that the ERACs are formed through aggregation of aberrant transmembrane proteins and work as the accumulation sites of multiple ERAC-forming proteins for their sequestration.
\end{abstract}

Key words: protein aggregation, organelle, unfolded protein response, ER stress, 4-PBA

\section{Introduction}

The endoplasmic reticulum (ER) is a membrane-bound cellular compartment in which newly synthesized secretory and transmembrane proteins are folded before being transported to the cell surface or other organelles. As exemplified by the vertebrate chloride channel cystic fibrosis transmembrane conductance regulator (CFTR) and its mutants, misfolded ER-client proteins are retained in the ER and are thus unable to work normally (Cheng et al., 1990). Moreover, misfolded proteins per se are harmful to cells when abundantly accumulated in the ER lumen (Umebayashi et al., 1997). Dysfunctional situations in the ER are tightly linked to ER accumulation of misfolded proteins and is collectively called ER stress.

\footnotetext{
$\dagger$ Both authors contributed equally to this work.

*To whom correspondence should be addressed: Yukio Kimata, Division of Biological Science, Graduated School of Science and Technology, Nara Institute of Science and Technology, 8916-5 Takayama, Ikoma, Nara 630-0192, Japan.

E-mail: kimata@bs.naist.jp

Abbreviations: 4-PBA, 4-phenyl butyric acid; carboxy-DCFDA, 5(and-6-)-carboxy-2',7'-dichlorofluorescein diacetate; CFTR, cystic fibrosis transmembrane conductance regulator; DTT, dithiothreitol; ER, endoplasmic reticulum; ERAC, ER-associated compartment; HA, hemagglutinin; PAGE, poly-acrylamide gel electrophoresis; PI, propidium iodide; SD, synthetic dextrose; TCA, trichloroacetic acid; UPR, unfolded protein response; YGRC, Yeast Genetic Resource Center.
}

The unfolded protein response (UPR) is a cytoprotective gene-induction program that triggered upon ER stress (Ron and Walter, 2007; Mori, 2009). It is widely believed that in Saccharomyces cerevisiae (hereafter simply called yeast) cells, the ER-located transmembrane protein Irel acts as a sole ER-stress sensor that evokes the UPR. Ire1 senses ER stress via multiple mechanisms (Kimata et al., 2007; Kimata and Kohno, 2011; Ishiwata-Kimata et al., 2018), and works as an endoribonuclease that promotes splicing of the HAC1 mRNA. The spliced form of the HAC1 mRNA is translated into a nuclear transcription-factor protein that is responsible for transcriptional induction in the UPR.

Misfolded proteins often form foci in cells, possibly in order to be sequestered from other cellular components. For instance, according to Escusa-Toret et al. (2013), misfolded cytosolic proteins are accumulated on the ER surface to form puncta called the Q bodies, the formation of which is thought to be beneficial for yeast cells. Some previous publications have reported that misfolded multimembranespanning proteins form ER-associated punctate structures, which is called the ER-associated compartments (ERACs), in yeast cells (Huyer et al., 2004; Liu et al., 2006; Fu and Sztul, 2009; Kakoi et al., 2013). As well as mutant versions of yeast endogenous transmembrane proteins, such as Ste6, heterologously expressed CFTR forms the ERAC even when not carrying a mutation. 
While some previous publications addressed the nature of the ERACs (Huyer et al., 2004; Fu and Sztul, 2009; Kakoi et al., 2013), many questions remain unanswered about their physiological properties and relevance. For instance, is the ERAC formation beneficial for cells? Are different ERAC-forming proteins accumulated in the same ERACs? Moreover, the molecular mechanism for ERAC formation is still largely unknown.

For better understanding of the ERACs, in this study, we explore yeast cells expressing a mutant form of Pma1, which is a plasma membrane-located multimembranespanning protein that acts as a proton pump when not carrying a mutation (Serrano et al., 1986). This mutant Pma1, which we named as Pma1-2308, formed the ERACs and induced the UPR when highly expressed in yeast cells. Cellular treatment with the chemical chaperone 4-phenyl butyric acid (4-PBA) inhibited the ERAC formation of Pma1-2308, leading to aggravation of ER stress caused by this mutant protein. This observation argues for a beneficial aspect of the ERACs.

\section{Materials and Methods}

\section{Plasmid construction}

Plasmid pTH761-CEN-mCherry_v4 contains a yeast codonoptimized version of the mCherry cDNA (Chu et al., 2014). The human CFTR cDNA was a gift from Prof. Kenji Kohno (Nara Inst. Sci. Tech.). Using these cDNAs as templates, we obtained the mCherry-coding and the CFTR-coding DNA fragments through high-fidelity PCR with Pyrobest DNA polymerase (Takara). The Pma1-coding DNA fragment was PCR-amplified from a yeast genome using conventional Takara ExTaq DNA polymerase.

The yeast centromeric plasmid pPM28 (URA3 marker; Merksamer et al., 2008) was used for expression of eroGFP under control of the TDH3 plasmid. After NheI/XbaI digestion, which resulted in excision of the eroGFP-coding sequence, pPM28 was ligated with the Pmal-coding and the mCherry-coding DNA fragments or the CFTR-coding and the mCherry-coding DNA fragments. The resulting plasmids, pYT-TDH3p-PMA1-mCherry and pYT-TDH3p-CFTR-mCherry, respectively, were used for expression of wild-type Pmal-mCherry and CFTR-mCherry in yeast cells. Due to PCR error, we also obtained the PMA1-2308 mutant version of pYT-TDH3p-PMA1-mCherry, which is named pYTTDH3p-PMA1-2308-mCherry. The CUP1 promoter sequence was PCR-amplified from the yeast genome and was substituted on the TDH3 promoter of pYT-TDH3p-PMA1-2308-mCherry for generation of pYT-CUP1p-PMA1-2308-mCherry, which was used for expression of Pma1-2308-mCherry under the control of the CUP1 promoter in yeast cells. The 13-tandem-copy Myc epitope-coding sequence was PCR-amplified from the plasmid pFA6a-13MyckanMX6 (Bahler et al., 1998), and was substituted on the mCherry-coding sequence of pYT-CUP1p-PMA1-2308-mCherry for generation of pYT-CUP1p-PMA1-2308-Myc, which was used for expression of Pma1-2308-Myc under the control of the CUP1 promoter in yeast cells. These plasmids were deposited in the Yeast Genetic Resource Center (YGRC), and the plasmid sequence information is available in our dropbox site (https:// www.dropbox.com/sh/ocqptzlcfafq7t0/AACA61a-YeaTkJSigzm JbUhwa?dl=0) and on the YGRC website (http://yeast.nig.ac.jp/ yeast/top.xhtml). For the empty vector control, pRS316 (Sikorski and Hieter, 1989) was employed.

The hemagglutinin (HA) epitope-tagged Ire1-gene centromeric (single-copy) plasmid pRS315-IRE1-HA, IRE1-gene centromeric (single-copy) plasmid pRS313-IRE1 and the empty vector pRS313 (HIS3 marker) were described in Kimata et al. (2004) and Sikorski and Hieter (1989).

\section{Yeast strains}

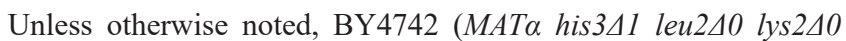
ura3 $\triangle 0$ ) was used as a wild-type strain and transformed with the aforementioned plasmids. In the experiments using 4-PBA, KMY1516 (MATa his3 leu2 lys2 ura3 trp1 ire1::TRP1 leu2::UPRE-GFP::LEU2 lys2::UPRE-lacZ::LYS2; Kimata et al., 2004) carrying pRS313-IRE1 was employed as a wild-type strain and transformed with the aforementioned plasmids, because the viability of BY4742 was somewhat sensitive to 4-PBA (data not shown). For the IRE1 gene-knockout experiments, KMY1516 cells transformed with pRS313-IRE1 and pRS313, respectively, were used as the IRE1+ and the ire1 $\triangle$ strains. For cellular expression of Ire1-HA, we employed KMY1015 (MATa his3 leu2 lys2 ura3 trp1 ire1::TRP1; Mori et al., 1996) transformed with pRS315-IRE1-HA.

Transformation of the BY4742 cells with PvuI-digested pYTCUP1p-PMA1-2308-mCherry together with NotI-digested pRS315 (LEU2 marker; Sikorski and Hieter, 1989) yielded the LEU2-marker version of pYT-CUP1p-PMA1-2308-mCherry via in vivo homologous recombination. The resulting transformants were again transformed with pPM28 in order to obtain cells producing both Pma1-2308-mCherry and eroGFP.

BY4742-derived HRD1/DOA10-double knockout strain (hrd1::kanMX4 doa10::SpHIS5MX) has been described previously (Mai et al., 2018), and here was transformed with pYTTDH3p-CFTR-mCherry. Alternatively, this HRD1/DOA10-double knockout strain was modified to carry both the LEU2-marker version of pYT-CUP1p-PMA1-2308-mCherry and the mCherry-toGFP replaced version of pTDH3p-CFTR-mCherry, which were also generated through in vivo homologous recombination.

Yeast transformation was performed using the standard lithiumacetate-based protocol (Kaiser et al., 1994).

\section{Yeast culture}

Unless otherwise noted, yeast cells were aerobically and exponentially shaken in synthetic dextrose (SD) medium containing 2\% glucose, $0.66 \%$ Difco Yeast Nitrogen Base (w/o amino acid) and appropriate auxotrophic requirements at $30^{\circ} \mathrm{C}$. Alternatively, cells 
were cultured at $23^{\circ} \mathrm{C}$ to obtain brighter CFTR-mCherry or CFTR-GFP signals. SD medium plates containing 2\% agar were incubated at $30^{\circ} \mathrm{C}$ after inoculation of yeast cells. For chemical treatment of cells, tunicamycin (Sigma-Aldrich; $2 \mathrm{mg} / \mathrm{mL}$ in dimethyl sulfoxide for stock solution), $\mathrm{CuSO}_{4}(0.5 \mathrm{M}$ in water for stock solution), and 4-PBA (Sigma-Aldrich; $0.5 \mathrm{M}$ in water for stock solution) were added into cultures, which were further shaken at $30^{\circ} \mathrm{C}$.

\section{Protein and RNA analyzes}

As described previously (Kimata et al., 2003; Mai et al., 2018), cells were lysed in the presence of $1 \%$ triton X-100 and analyzed by Western blotting using mouse monoclonal antibodies $12 \mathrm{CA} 5$ (anti-HA epitope; Roche) and 9E10 (anti-c-Myc epitope anti-HA antibody (anti-c-Myc; Roche). Cell lysates were centrifuged at $15,000 \times g$ for $30 \mathrm{~min}$ to obtain pellet fractions. In order to denature proteins, we added $10 \%$ trichloroacetic acid (TCA) and 100 $\mathrm{mM}$ dithiothreitol (DTT) into the SDS poly-acrylamide gel electrophoresis (SDS-PADE) loading buffer.

Splicing of the HACl mRNA was monitored by RT-PCR amplification of the $H A C l$ species from total RNA samples, which was followed by standard agarose-gel electrophoresis (Promlek et al., 2011; Mai et al., 2018). The ethidium bromide-fluorescence images of the resulting gels was used for image analysis which was followed by calculation of "the HACl mRNA-splicing efficiency" using the formula $\left[100 \times\left(\right.\right.$ band intensity of $\left.H A C 1^{\mathrm{i}}\right) /\{$ (band intensity of $\left.H A C 1^{\mathrm{i}}\right)+\left(\right.$ band intensity of $\left.\left.H A C 1^{\mathrm{u}}\right)\right\}$ ], where $H A C 1^{\mathrm{i}}$ and $H A C l^{\mathrm{u}}$, respectively, are the spliced and unspliced forms of the HAC1 mRNA.

\section{Microscopic observation}

In order to stain cells with 5-(and-6-)-carboxy-2',7'dichlorofluorescein diacetate (carboxy-DCFDA), cells were suspended in sodium citrate buffer $(\mathrm{pH} 5.0)$ containing $2 \%$ glucose and $10 \mathrm{mM}$ carboxyl-DCFDA for $30 \mathrm{~min}$.

We observed yeast cells under the Keyence BZ-9000E microscope and the objective lens CFI Plan Apo $\lambda 100 \mathrm{xH}$ (Nikon). GFP and carboxy-DCFDA fluorescence was observed using a preset filter set (excitation wavelength, 470/30; dichroic mirror wavelength, 495; emission, 535/25). The mCherry fluorescence was observed using another preset filter set (excitation wavelength, 560/20; dichroic mirror wavelength, 595; emission, 630/30). The exposure time was 0.5 or $1.0 \mathrm{sec}$ for wild-type Pma1-mCherry or Pma1-2308-mCherry fluorescence, $1.0 \mathrm{sec}$ for eroGFP fluorescence, $8 \mathrm{sec}$ for CFTR-mCherry fluorescence and $0.8 \mathrm{sec}$ for carboxy-DCFDA fluorescence.

In order to quantitatively express ER dispersion of Pma1-2308mCherry, we counted cells showing nuclear-ER unbroken ringlike fluorescent image of Pma1-2308-mCherry. More than 50 fluorescence-emitting cells were checked per sample, and its portion against total fluorescence-emitting cells are expressed as the mean of three independent cultures.

\section{Flow cytometry}

The flow cytometer Accuri C6 Plus (BD Biosciences) were operated under the following conditions: flow rate, slow; threshold, FCS-H $>80,000$; count cell number, 20,000. In order to count propidium iodide (PI)-stainable cells, cultures were mixed with PI (10 $\mu \mathrm{g} / \mathrm{mL}$ (final concentration)), further incubated for $30 \mathrm{~min}$ and subjected to flow cytometry.

\section{Statistical analysis}

Numerical data were obtained through multiple (basically three) determinations using independent clones, and were evaluated by the unpaired (homoscedastic) two-tailed t-test.

\section{Results}

At the beginning of this study, we constructed a yeast expression plasmid (pYT- TDH3p-PMA1-mCherry) to produce a C-terminally mCherry-tagged version of Pma1 (Pma1-mCherry) under the control of the strong and constitutive $T D H 3$ promoter. As shown in Fig. 1A and Fig. S1, mCherry fluorescence was observed in the cell periphery and the vacuoles, which were stained by the vacuolestaining dye carboxy-DCFDA, of yeast cells harboring this plasmid. This florescence pattern is consistent with that reported by Eastwood and Meneghini (2015) and suggests that Pma1-mCherry on the plasma membrane was partly subjected to endocytosis and transported to the vacuoles.

Since we yielded the PMA1 gene through PCR in which the DNA replication fidelity was low (see the Materials and Methods section) to construct pYT-TDH3p-PMA1mCherry, we also obtained a PMA1 gene-mutant version of this plasmid. Based on our further analysis described in the next paragraph, we named this mutant as PMA1-2308. Expression of Pma1-2308 is likely to harm cells since yeast cells transformed with the PMA1-2308 mutant variant of pYT-TDH3p-PMA1-mCherry (pYT-TDH3p-PMA1-2308mCherry) proliferated very slowly (Fig. S2). Intriguingly, unlike wild-type Pmal-mCherry, Pma1-2308-mCherry exhibited a punctate distribution pattern, which did not merge with the vacuole marker, in yeast cells (Fig. 1A and Fig. S1).

The PMA1-2308 mutant carried one silent (non-amino acid-replacing) nucleotide replacement (Thymine1548-toCytosine) and four non-silent (amino acid-replacing) nucleotide replacements which are indicated in Fig. S3A (the nucleotides are numbered from the initiation codon of the PMAl gene). In order to determine which is responsible for the punctum-forming phenotype of the PMA1-2308 mutation, we introduced each of the non-silent nucleotide replacements into the wild-type Pmal-mCherry gene. As shown in Fig. S3A, Pma1-mCherry exhibited a punctate distribution when carrying the Thymine 2308-to-Cytosine 
(A) Wild-type Pma1-mCherry (TEF1 promoter)
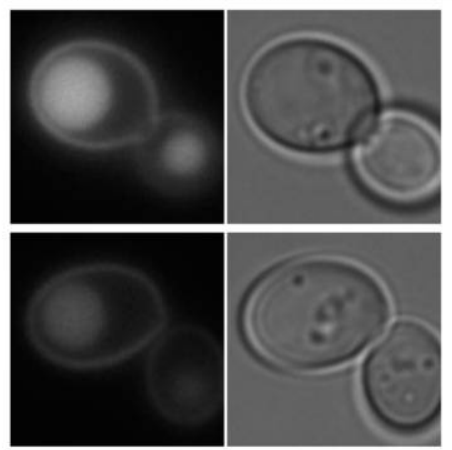

Pma1-2308-mCherry (TEF1 promoter)
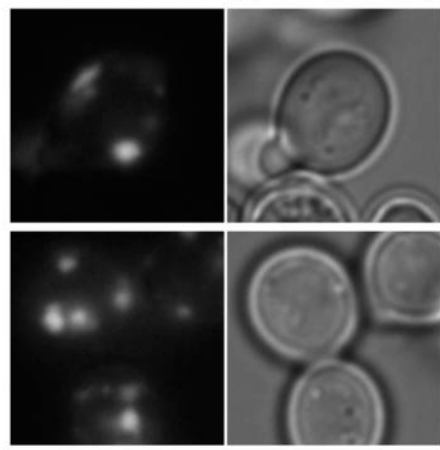

(B)
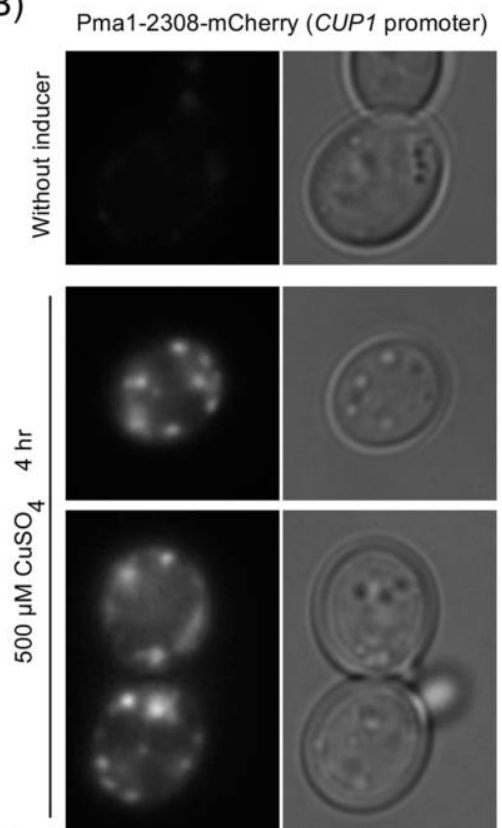

(C)

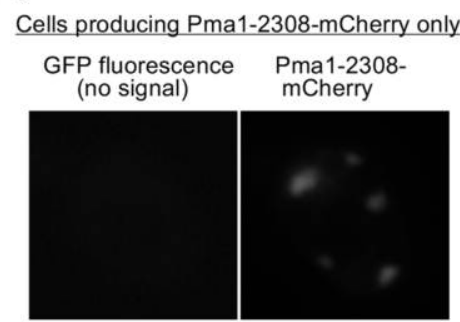

Cells producing eroGFP only
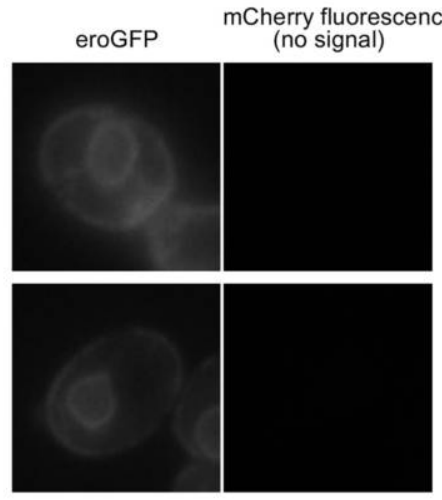

Cells producing both eroGFP and Pma1-2308-mCherry
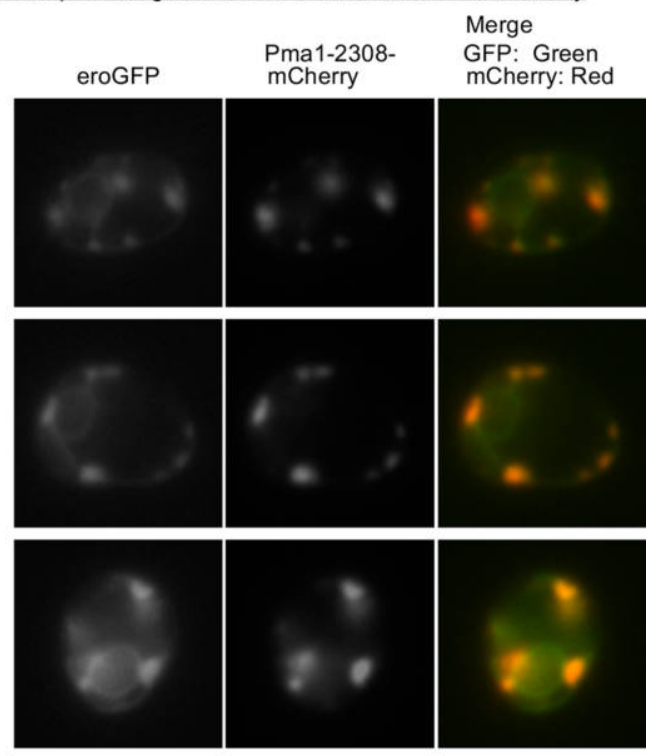

$5 \mu \mathrm{m}$

Fig. 1. Pma1-2308 forms the ERACs. (A) Wild-type cells producing wild-type Pma1-mCherry or Pma1-2308-mCherry under TDH3-promoter control were observed under fluorescence microscopy. (B) Wild-type cells that produce Pma1-2308-mCherry under CUP1-promoter control were cultured with or without $500 \mu \mathrm{M} \mathrm{CuSO}_{4}$ for $4 \mathrm{hr}$ and observed under fluorescence microscopy. (C) Wild-type cells that produce both (or either) Pma1-2308-mCherry (under CUP1-promoter control) and eroGFP (under $T D H 3$-promoter control) were cultured with $500 \mu \mathrm{M} \mathrm{CuSO}_{4}$ for 4 hr and observed under fluorescent microscopy. 


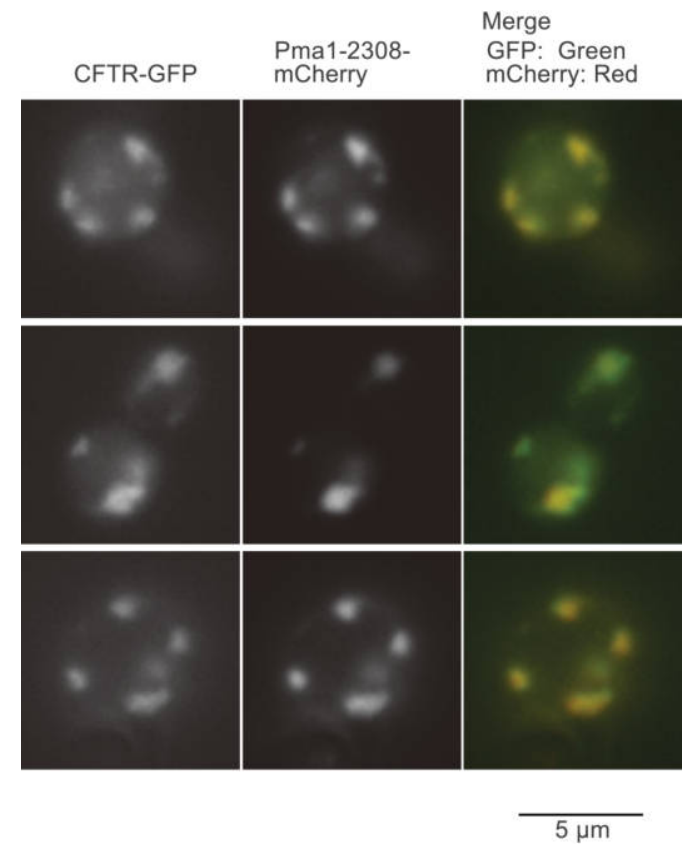

Fig. 2. Pma1-2308 and CFTR co-localize in the same ERACs. hrd1 $\triangle$ doa104 cells that produce both Pma1-2308-mCherry (under CUP1promoter control) and CFTR-GFP (under TDH3-promoter control) were cultured with $500 \mu \mathrm{M} \mathrm{CuSO}_{4}$ for $4 \mathrm{hr}$ and observed under fluorescent microscopy.

single mutation. It should be also noted that the Thymine 2308-to-Cytosine single replacement was also responsible for the growth-retardation phenotype of the PMA1-2308 mutation (Fig. S3B).

In order to grow yeast cells carrying the PMA1-2308 gene to an extent that sufficiently allowed the belowmentioned experiments, we constructed yeast cells in which Pma1-mCherry-2308 was expressed from an inducible promoter. As expected, yeast cells transformed with pYTCUP1p-PMA1-2308-mCherry, from which expression of Pma-2308-mCherry was regulated under the control of the copper ion-inducible CUP1 promoter, exhibited fluorescence when cultured in the presence of $500 \mu \mathrm{M} \mathrm{CuSO}_{4}$ (Fig. 1B). According to our flowcytometric analysis (Fig. S4), the expression level of Pma1-2308-mCherry reached a maximum level 4-hr after induction onset under our experimental condition.

In the experiment shown in Fig. S5, the C-terminally Myc-epitope-tagged versions of wild-type Pmal (wild-type Pma1-Myc) and Pma1-2308 (Pma1-2308-Myc) were expressed in wild-type cells, the lysates of which were analyzed by anti-Myc Western blotting. Although protein samples were denatured by SDS, DTT and TCA before electrophoresis (see the Materials and Methods section), Pma1-2308-Myc, as well as wild-type Pma1-Myc, appeared as slightly laddered and smeared bands for unknown reason(s) (Fig. S5). We next checked stability of wild-type Pma1-Myc and Pma1-2308-Myc using the cycloheximide-chase technique. According to our data shown in Fig. S6, Pma1-2308-Myc seemed to be fairly stable (more stable than wild-type Pma1-Myc) in yeast cells.

In the experiment shown in Fig. 1C, we observed yeast cells expressing both (or either) Pma1-2308-mCherry and eroGFP, which is an ER-localized variant of GFP (Merksamer et al., 2008). When expressed alone in yeast cells, eroGFP showed a typical double-ring ER distribution pattern. Meanwhile, eroGFP was also distributed to punctate dots that were located on or adjacent to the double rings when it was expressed together with Pma1-2308mCherry. It should be also noted that Pma1-2308-mCherry was located on the eroGFP puncta. We thus deduce that Pma1-2308-mCherry forms and is accumulated in the ERACs, as described in the Discussion section.

As described in the Introduction section, heterologously expressed CFTR is also located in the ERACs in yeast cells. In order to observe intracellular localization of Cterminally GFP-tagged CFTR (CFTR-GFP), we employed here the hrd14/doal04 double deletion mutant strain because CFTR-GFP is quickly degraded through the ERassociated protein degradation in a manner dependent on the E3 enzymes Hrd1 and Doa10 (Gnann et al., 2004) and hardly emits observable fluorescence in wild-type yeast cells. As shown in Fig. 2, CFTR-GFP and Pma1-2308mCherry were co-localized, indicating that these two different ERAC-forming proteins are accumulated in the same ERACs.

Next, we asked if cellular expression of Pma1-2308mCherry induces ER stress that triggers the UPR, which is monitorable through the $H A C 1$ mRNA splicing. As described previously (Promlek et al., 2011; Mai et al., 2018), here we amplified the $\mathrm{HACl}$ spices from total RNA samples using the reverse transcription (RT)-PCR technique. Fig. 3A shows that Pma1-2308-mCherry, but not wild-type Pma1-mCherry, triggered HAC1-mRNA splicing when it was expressed from the TDH3 promoter. According to our data shown in Fig. S7, the Thymine 2308-to-Cytosine single replacement was responsible for the ER-stressing phenotype of the PMA1-2308 mutation.

UPR induction by the Pma1-2308-mCherry expressed from the CUP1 promoter is described later in this paper. In the experiment shown in Fig. 3B, cells were grown on agar media containing or not containing an inducible concentration of $\mathrm{CuSO}_{4}$. Cells carrying the CUP1 promotercontrolled Pma1-2308-mCherry-expression plasmid showed growth retardation, which was aggravated by the absence of the IREI gene, on the inducer-containing plate. These observations indicate that, at least partly, Pma1-2308-mCherry harms yeast cells through induction of ER stress. Fig. S8 exhibits the ERAC formation of Pma1-2308-mCherry in irel 14 cells.

Since it is widely accepted that 4-PBA is a chemical chaperone that inhibits protein aggregation (Kubota et al., 
(A)

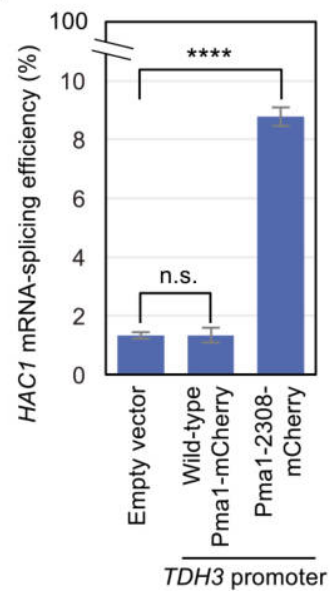

(B)

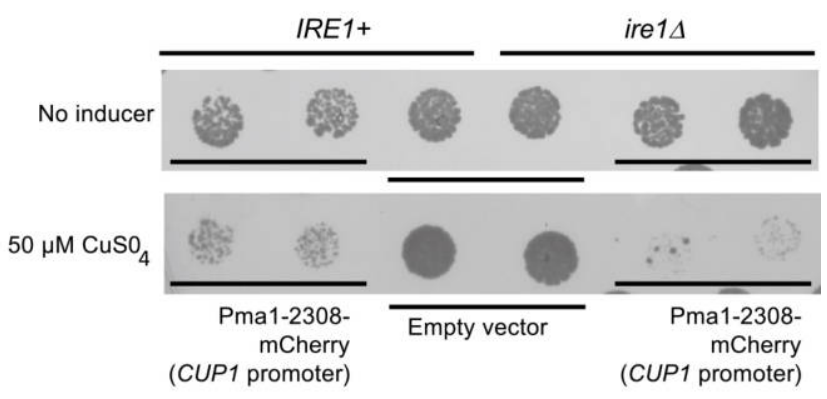

Fig. 3. Pma1-2308 induces the UPR. (A) Wild-type cells producing either (or neither) wild-type Pma1-mCherry or Pma1-2308-mCherry under the TDH3-promoter control were checked for HAC1 mRNA-splicing efficiency. ****: $\mathrm{p}<0.001$, n.s.: not significant (p>0.05). (B) IRE1+ and ire1A cells carrying the $C U P 1$ promoter-controlled Pma1-2308-mCherry expression plasmid or the empty vector were spotted on SD agar plates supplemented or not with $50 \mu \mathrm{M} \mathrm{CuSO}_{4}$, which were then incubated for 3 days. On each spot, cells equivalent to $\mathrm{OD}_{600}=0.00015$ were inoculated.

2006; Kolb et al., 2015), we asked if 4-PBA affects the cellular localization of ERAC-forming proteins. As shown in Fig. 4A and 4B, treatment of cells with $1 \mathrm{mM} 4-\mathrm{PBA}$ caused dispersed distribution of Pma1-2308-mCherry and C-terminally mCherry-tagged CFTR (CFTR-mCherry). Since the cellular distribution of Pma1-2308-mCherry and CFTR-mCherry in 4-PBA-treated cells demonstrated a typical double-ring-like ER pattern, we deduce that, at least partly, they are retained in the ER without being transported to the cell surface.

In the experiment shown in Fig. 4C, lysates of cells producing Pma1-2308-Myc were subjected to anti-Myc Western blotting, indicating that treatment of cells with 4-PBA did not significantly change the cellular abundance of Pma1-2308-Myc (compare Lane 2 to Lane 1). We also subjected the total cell lysates to high-speed centrifugation and noticed that cellular treatment with 4-PBA decreased the abundance of Pma1-2308-Myc in the pellet fractions (compare Lane 4 to Lane 3).

Next, we asked if 4-PBA can disperse already-ERACforming Pma1-2308-mCherry proteins. In the experiment shown in Fig. 4D, yeast cells producing Pma1-2308mCherry from the CUP1 promoter through 4-hr culture with $500 \mu \mathrm{M} \mathrm{CuSO}_{4}$ were washed with normal medium and further cultured in the absence of an inducible concentration of $\mathrm{CuSO}_{4}$ (chase culturing). We then found that Pma1-2308-mCherry was dispersed, though not completely, when chase culturing was performed in the presence of $1 \mathrm{mM}$ 4-PBA. In order to express this observation quantitatively, we counted cells showing dispersed distribution of Pma1-2308-mCherry, which was represented by its nuclear-ER unbroken ring-like image (Fig. 4D).

In the final part of this study, we asked if ER stress is alleviated or aggravated when the ERAC formation of Pma1-2308-mCherry is compromised by cellular treatment with 4-PBA. According to our previous publication (Mai et al., 2018), the cellular abundance of C-terminally HA epitope-tagged Ire1 (Ire1-HA) was drastically decreased by treatment of yeast cells with $5 \mathrm{mM}$ 4-PBA. However, such was not observed, and the cellular Ire1-HA abundance was almost unchanged when yeast cells were cultured in the presence of $1 \mathrm{mM} 4$-PBA for $1 \mathrm{hr}$ (Fig. 5A). In order to monitor the effect of 4-PBA on the cellular UPR level, we thus employed the experimental condition represented in Fig. 5B, in which yeast cells were treated with $1 \mathrm{mM} \mathrm{4-}$ PBA for no longer than $1 \mathrm{hr}$ (4-PBA was added 3-hr after addition of $500 \mu \mathrm{M} \mathrm{CuSO}$ into culture). Fig. $5 \mathrm{C}$ shows that, under this condition, Pma1-2308-mCherry was partially dispersed. Notably, 4-PBA further increased the UPR level that was induced by Pma1-2308-mCherry, at least under the experimental procedure employed here (Fig. 5D). We therefore deduce that the dispersion of Pma1-2308mCherry by 4-PBA aggravates ER stress. This observation is in contrast with the case of stressing yeast cells with the canonical ER stressor tunicamycin, in which 4-PBA compromised the UPR (Fig. S9). In the experiments shown in Fig. 5, we avoided treatment of yeast cells with 4-PBA for a longer duration, which decreased the cellular Ire1 abundance (data not shown), possibly leading to a reduction of the HACl-mRNA level and to an underestimation of the cellular ER-stress level. PI is known to stain dead yeast cells (Kwolek-Mirek and Zadrag-Tecza, 2014). In the experiment shown in Fig. 5E, we measured the portion of PI-stainable cells in cultures and found that the cellular expression of Pma1-2308-mCherry caused a reduction of cell viability, which was aggravated by 4-PBA. 
(A)

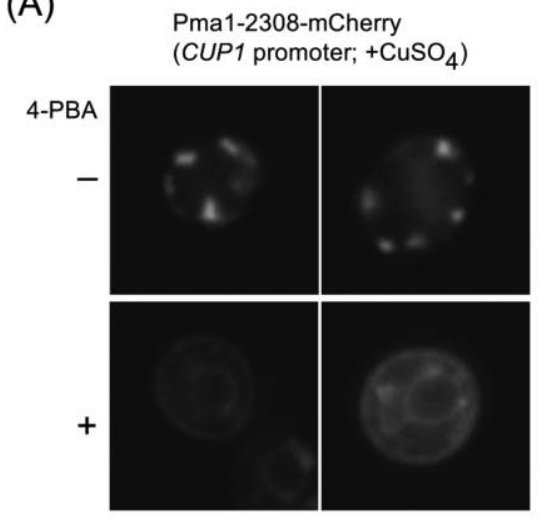

(B)

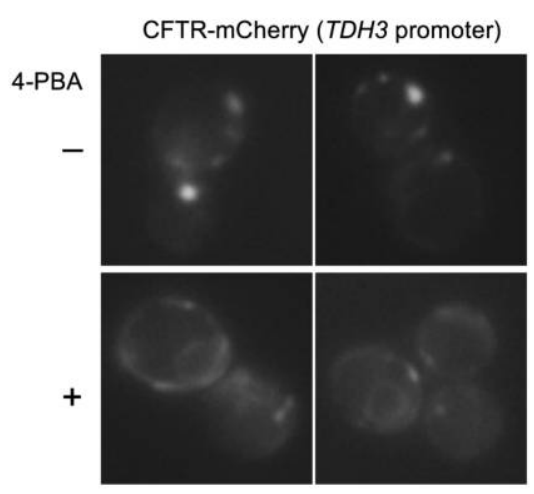

(C)

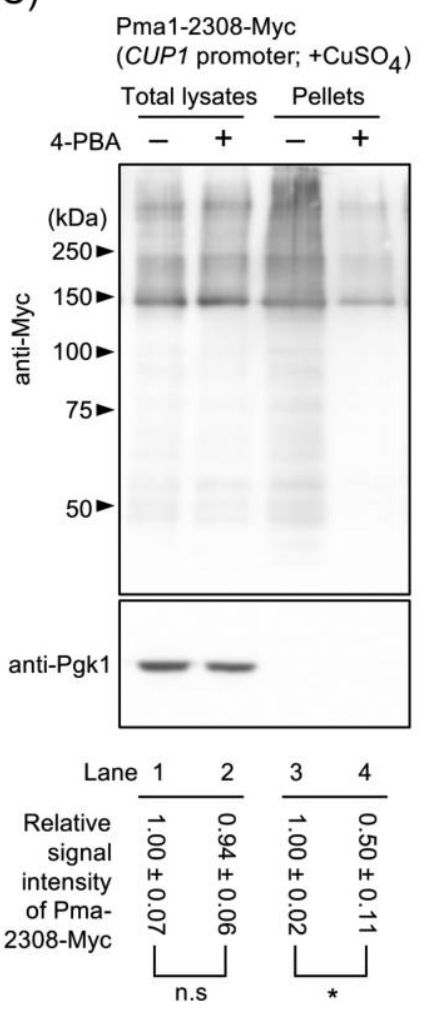

(D)

Pma1-2308-mCherry (CUP1 promoter)

0-min after $\mathrm{CuSO}_{4}$ washout

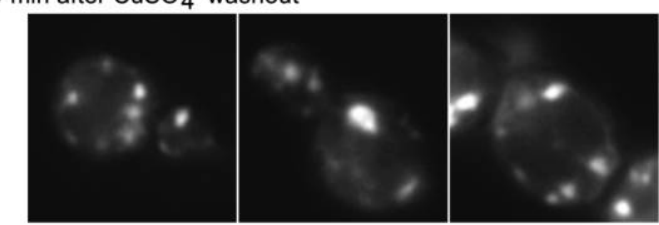

150-min after $\mathrm{CuSO}_{4}$ washout (Chase culture without 4-PBA)

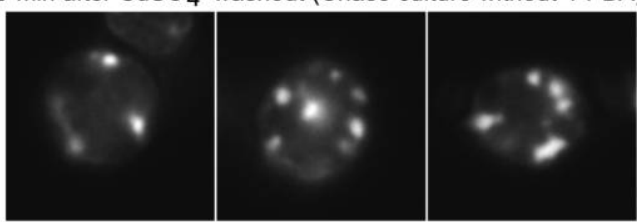

nuclear-ER

ring-like

fluorescence

image

$11.7 \pm 5.8 \%$

150-min after $\mathrm{CuSO}_{4}$ washout (Chase culture with 4-PBA)

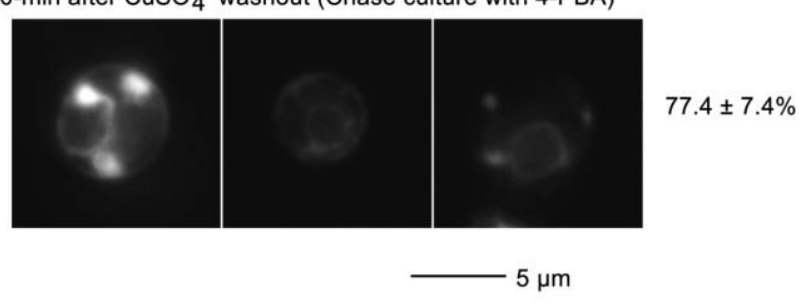

Fig. 4. 4-PBA compromises the ERAC formation. (A) Wild-type cells that produce Pma1-2308-mCherry under CUP1-promoter control were cultured with $500 \mu \mathrm{M} \mathrm{CuSO}_{4}$ and $1 \mathrm{mM}$ 4-PBA (or with $500 \mu \mathrm{M} \mathrm{CuSO}_{4}$ only) for $4 \mathrm{hr}$ and observed under fluorescence microscopy. (B) hrd1 $\Delta$ doal0 4 cells producing CFTR-mCherry under TDH3-promoter control were cultured with or without $500 \mu \mathrm{M} 4$-PBA for 3 hr and observed under fluorescence microscopy. (C) Wild-type cells that produce Pma1-2308-Myc under CUP1-promoter control were cultured with $500 \mu \mathrm{M} \mathrm{CuSO}_{4}$ and $1 \mathrm{mM} 4-\mathrm{PBA}$ (or with $500 \mu \mathrm{M} \mathrm{CuSO}_{4}$ only) for $4 \mathrm{hr}$ and lysed in the presence of $1 \%$ Triton-X100. The total cell lysates were then separated by high-speed centrifugation and analyzed by anti-Myc Western blotting. Each lane contained sample from cells equivalent to $\mathrm{OD}_{600}=0.13$ for "total lysates" and from cells equivalent to $\mathrm{OD}_{600}=2.5$ for "pellets." Anti-Pgk1 Western blot served as a loading control. *: $\mathrm{p}<0.05$, n.s.: not significant (p $>0.05$ ). (D) After being cultured in the presence of $500 \mu \mathrm{M} \mathrm{CuSO}_{4}$, cells producing Pma1-2308-mCherry under CUP1-promoter control were washed four times with SD medium (CuSO washout) and further incubated in the presence or absence of $1 \mathrm{mM} 4-\mathrm{PBA}$ for $150 \mathrm{~min}$ (chase culture).

\section{Discussion}

To our knowledge, the ERAC formation in yeast cells was initially reported by Huyer et al. (2004). The ERACs are defined as ER subcompartments in which misfolded multimembrane-spanning proteins are localized. The ER is a flat-shaped membranous sac, which, according to the electron microscopic images reported by Huyer et al. 
(A)

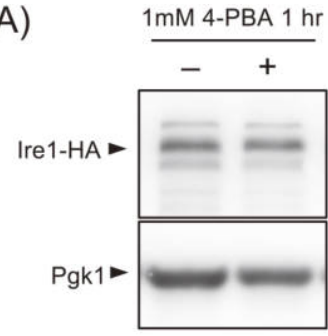

(C)

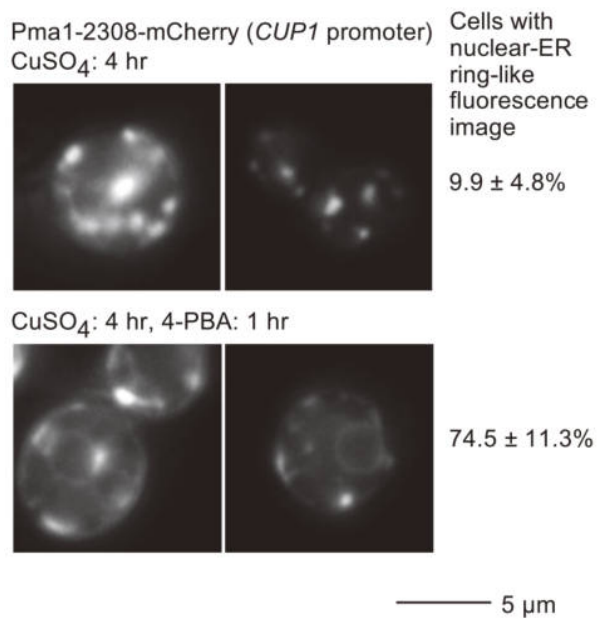

(B)

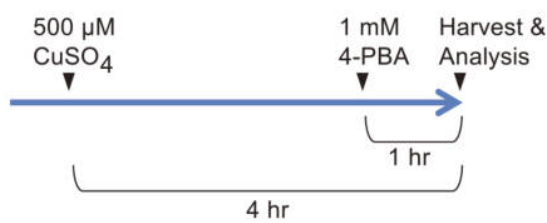

(D)

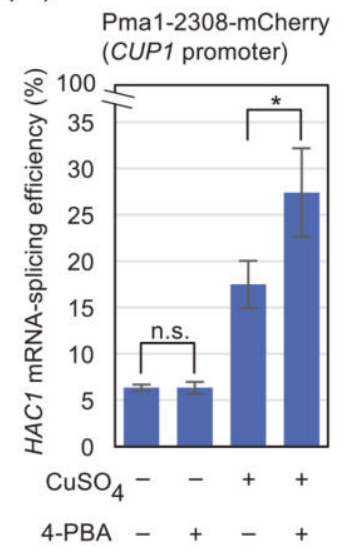

(E)

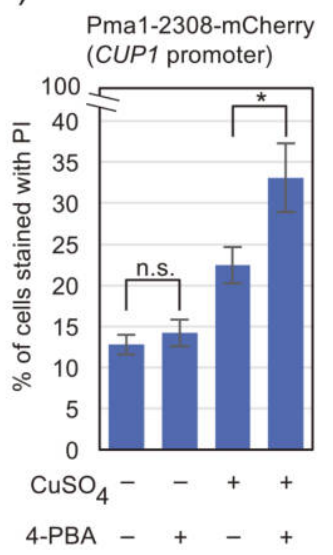

Fig. 5. 4-PBA enhances the UPR induced by Pma1-2308-mCherry. (A) Cells producing Ire1-HA were treated with 1 mM 4-PBA for 1 hr or left untreated, and the total lysates from cells equivalent to $\mathrm{OD}_{600}=0.13$ were analyzed by anti-HA Western blotting. Anti-Pgk1 Western blot served as a loading control. (B) Procedure for treatment of cells with 4-PBA 3 hr-after the induction onset of Pma1-2308-mCherry expression. (C) Cells that produce Pma1-2308-mCherry under CUP1-promoter control were treated as shown in panel B, and were observed under fluorescence microscopy. (D) and (E) Cells that produce Pma1-2308-mCherry under CUP1-promoter control were treated as shown in panel B (or cultured with either or none of the chemicals), and were checked for the HAC1-mRNA splicing (D) or the portion of PI-stainable cells in the cultures (E). *: $\mathrm{p}<0.05$, n.s.: not significant ( $\mathrm{p}>0.05)$.

(2004) and Fu and Sztul (2009), is partly folded and stacked to form multiple layers on the site where the ERACs are formed. This observation is consistent with our fluorescence microscopy images of yeast cells producing both eroGFP, which is thought to be diffusively located in the ER, and Pma1-2308-mCherry (Fig. 1C). In yeast cells producing both fluorescent proteins, eroGFP fluorescence displayed faint double rings and bright puncta adjacent to the rings, and Pma1-2308-mCherry was located on the eroGFP puncta. It should be also noted that Pma1-2308mCherry and heterologously expressed CFTR were colocalized (Fig. 2). To our knowledge, this is the first report showing that two different ERAC-forming proteins are accumulated in the same ERACs.

As described in Kubota et al. (2006), 4-PBA is likely to function as a chemical chaperone that prevents aggregation of denatured proteins. Moreover, 4-PBA suppresses the UPR in mammalian and yeast cells, and is thought to be a promising chemical that can be used for therapeutic purposes for various diseases caused by ER accumulation of misfolded proteins (Kubota et al., 2006; Kolb et al., 2015).
However, we reported previously that at least in yeast cells, 4-PBA compromises the UPR not via restoration of the protein folding status in the ER but via reduction of the cellular Ire1 abundance. Meanwhile, here we employed a lower concentration of 4-PBA than that was used in previous publications by us and others (Kubota et al., 2006; Mai et al., 2018), and found that 4-PBA suppresses the tunicamycin-induced UPR without reduction of the cellular Ire1 abundance (Fig. 5A and Fig. S9). Intriguingly, 4-PBA extinguished the ERACs and caused a dispersed distribution of ERAC-forming proteins across the ER (Fig. 4). Therefore, we conclude that 4-PBA can actually work as a molecular chaperone in yeast cells.

In the experiment shown in Fig. 4C, we separated cell lysates through high-speed centrifugation, and found that the amount of Pma1-2308-Myc in the pellet fraction was decreased by cellular treatment with 4-PBA. We deduce that accumulation of Pma1-2308-Myc in the pellet fraction, which was compromised by 4-PBA, represents its aggregation. Taken together, our observations support the notion that the ERACs are formed through aggregation of ERAC- 
forming proteins. In agreement with this idea, the fluorescence-recovery-after-photobleaching analysis performed by Fu et al. (2009) showed that in yeast cells, the CFTR becomes less mobile when accumulated into the ERACs.

Unlike other ERAC-forming proteins (Kakoi et al., 2013 and our unpublished data), cellular expression of Pma1-2308-mCherry clearly induced the UPR in yeast cells (Fig. 3A and Fig. 5D), indicating that Pma1-2308-mCherry damages the ER. On the other hand, our data showing in Fig. S8 demonstrates that the UPR signaling pathway is dispensable for the ERAC formation of Pma1-2308mCherry. Meanwhile, cytotoxicity of Pma1-2308-mCherry was enhanced by the IRE1-gene deletion (Fig. 3B). It is widely accepted that, in general, the IREl-gene deletion aggravates cellular damage caused by ER-stressing stimuli (Umebayashi et al., 1997; Kubota et al., 2006; Miyagawa et al., 2014; Le et al., 2016).

Intriguingly, the UPR induced by Pma1-2308-mCherry was aggravated by 4-PBA (Fig. 5D). Moreover, viability of cells expressing Pma1-2308-mCherry was decreased by 4PBA (Fig. 5E). Since, as described above, 4-PBA disperse the ERACs, we assume that these findings represent a beneficial aspect of the ERAC formation. Meanwhile, we cannot exclude a possibility that 4-PBA enhances the Pma1-2308-induced ER stress and its resulting cytotoxicity by another reason. In other words, 4-PBA may be potentially ER-stressing, and may cause a cellular damage which additively or synergistically induces ER stress and harms cells together with cellular expression of Pma1-2308.

Nevertheless, it should also be noted, at least in the case of other ER-stressing stimuli, including cellular treatment with tunicamycin, the UPR was attenuated by 4-PBA (Fig. S9; Kubota et al., 2006; Le et al., 2016). Moreover, according to Kubota et al. (2006), 4-PBA supports cellular growth of ire1 $\Delta$ cells in the presence of tunicamycin. These observations demonstrate an effect of 4-PBA to mitigate ER stress, and thus argue against the aforementioned idea that 4-PBA per se is potentially ER stressing.

In conclusion, in this study, we disclose some physiological properties of yeast ERACs. We propose that the ERACs are formed through aggregation of aberrant multimembrane-spanning proteins, and work as sequestration sites of multiple client proteins, which are harmful for cells when diffusively distributed across the ER. According to Kakoi et al. (2013), multiple cellular proteins, namely COPII-forming factors and cytosolic DnaJ-family proteins, are likely to contribute to the ERAC formation of CFTR. In agreement with our argument presented here, this insight suggests that yeast cells actively form the ERACs when aberrant multimembrane-spanning proteins are accumulated.

Acknowledgments. We deeply thank to Prof. Kenji Kohno and Prof. Hiroshi Takagi (Nara Inst. Sci. Tech.) for a wide variety of valuable supports, which have been indispensable for this study. This study is financially supported by Ohsumi Frontier Science Foundation to Y.K.

\section{References}

Bahler, J., Wu, J.Q., Longtine, M.S., Shah, N.G., McKenzie, A., 3rd, Steever, A.B., Wach, A., Philippsen, P., and Pringle, J.R. 1998. Heterologous modules for efficient and versatile PCR-based gene targeting in Schizosaccharomyces pombe. Yeast, 14: 943-951.

Cheng, S.H., Gregory, R.J., Marshall, J., Paul, S., Souza, D.W., White, G.A., O'Riordan, C.R., and Smith, A.E. 1990. Defective intracellular transport and processing of CFTR is the molecular basis of most cystic fibrosis. Cell, 63: 827-834.

Chu, D., Kazana, E., Bellanger, N., Singh, T., Tuite, M.F., and von der Haar, T. 2014. Translation elongation can control translation initiation on eukaryotic mRNAs. EMBO J., 33: 21-34.

Eastwood, M.D. and Meneghini, M.D. 2015. Developmental Coordination of Gamete Differentiation with Programmed Cell Death in Sporulating Yeast. Eukaryot. Cell, 14: 858-867.

Escusa-Toret, S., Vonk, W.I., and Frydman, J. 2013. Spatial sequestration of misfolded proteins by a dynamic chaperone pathway enhances cellular fitness during stress. Nat. Cell Biol., 15: 1231-1243.

Fu, L. and Sztul, E. 2009. ER-associated complexes (ERACs) containing aggregated cystic fibrosis transmembrane conductance regulator (CFTR) are degraded by autophagy. Eur. J. Cell Biol., 88: 215-226.

Gnann, A., Riordan, J.R., and Wolf, D.H. 2004. Cystic fibrosis transmembrane conductance regulator degradation depends on the lectins $\mathrm{Htm} 1 \mathrm{p} /$ EDEM and the Cdc48 protein complex in yeast. Mol. Biol. Cell, 15: $4125-4135$.

Huyer, G., Longsworth, G.L., Mason, D.L., Mallampalli, M.P., McCaffery, J.M., Wright, R.L., and Michaelis, S. 2004. A striking quality control subcompartment in Saccharomyces cerevisiae: the endoplasmic reticulum-associated compartment. Mol. Biol. Cell, 15: 908-921.

Ishiwata-Kimata, Y., Le, G.Q., and Kimata, Y. 2018. Stress-sensing and regulatory mechanism of the endoplasmic-stress sensors Ire1 and PERK. Endoplasmic Reticulum stress in Diseases, 5: 1-10.

Kaiser, C., Michaelis, S., and Mitchell, A. 1994. Methods in Yeast Genetics: A Cold Spring Harbor Laboratory Course Manual. Cold Spring Harbor Laboratory (New York).

Kakoi, S., Yorimitsu, T., and Sato, K. 2013. COPII machinery cooperates with ER-localized Hsp40 to sequester misfolded membrane proteins into ER-associated compartments. Mol. Biol. Cell, 24: 633-642.

Kimata, Y., Kimata, Y.I., Shimizu, Y., Abe, H., Farcasanu, I.C., Takeuchi, M., Rose, M.D., and Kohno, K. 2003. Genetic evidence for a role of $\mathrm{BiP} / \mathrm{Kar} 2$ that regulates Ire1 in response to accumulation of unfolded proteins. Mol. Biol. Cell, 14: 2559-2569.

Kimata, Y., Oikawa, D., Shimizu, Y., Ishiwata-Kimata, Y., and Kohno, K. 2004. A role for $\mathrm{BiP}$ as an adjustor for the endoplasmic reticulum stress-sensing protein Ire1. J. Cell Biol., 167: 445-456.

Kimata, Y., Ishiwata-Kimata, Y., Ito, T., Hirata, A., Suzuki, T., Oikawa, D., Takeuchi, M., and Kohno, K. 2007. Two regulatory steps of ERstress sensor Ire1 involving its cluster formation and interaction with unfolded proteins. J. Cell Biol., 179: 75-86.

Kimata, Y. and Kohno, K. 2011. Endoplasmic reticulum stress-sensing mechanisms in yeast and mammalian cells. Curr. Opin. Cell Biol., 23: 135-142.

Kolb, P.S., Ayaub, E.A., Zhou, W., Yum, V., Dickhout, J.G., and Ask, K. 2015. The therapeutic effects of 4-phenylbutyric acid in maintaining proteostasis. Int. J. Biochem. Cell Biol., 61: 45-52.

Kubota, K., Niinuma, Y., Kaneko, M., Okuma, Y., Sugai, M., Omura, T., Uesugi, M., Uehara, T., Hosoi, T., and Nomura, Y. 2006. Suppressive effects of 4-phenylbutyrate on the aggregation of Pael receptors and endoplasmic reticulum stress. J. Neurochem., 97: 1259-1268.

Kwolek-Mirek, M., and Zadrag-Tecza, R. 2014. Comparison of methods 
used for assessing the viability and vitality of yeast cells. FEMS Yeast Res., 14: 1068-1079.

Le, Q.G., Ishiwata-Kimata, Y., Kohno, K., and Kimata, Y. 2016. Cadmium impairs protein folding in the endoplasmic reticulum and induces the unfolded protein response. FEMS Yeast Res., 16: fow049.

Liu, Y., Sitaraman, S., and Chang, A. 2006. Multiple degradation pathways for misfolded mutants of the yeast plasma membrane ATPase, Pma1. J. Biol. Chem., 281: 31457-31466.

Mai, C.T., Le, Q.G., Ishiwata-Kimata, Y., Takagi, H., Kohno, K., and Kimata, Y. 2018. 4-Phenylbutyrate suppresses the unfolded protein response without restoring protein folding in Saccharomyces cerevisiae. FEMS Yeast Res., 18: foy 016.

Merksamer, P.I., Trusina, A., and Papa, F.R. 2008. Real-time redox measurements during endoplasmic reticulum stress reveal interlinked protein folding functions. Cell, 135: 933-947.

Miyagawa, K., Ishiwata-Kimata, Y., Kohno, K., and Kimata, Y. 2014. Ethanol stress impairs protein folding in the endoplasmic reticulum and activates Ire1 in Saccharomyces cerevisiae. Biosci. Biotechnol. Biochem., 78: 1389-1391.

Mori, K., Kawahara, T., Yoshida, H., Yanagi, H., and Yura, T. 1996. Signalling from endoplasmic reticulum to nucleus: transcription factor with a basic-leucine zipper motif is required for the unfolded protein- response pathway. Genes Cells, 1: 803-817.

Mori, K. 2009. Signalling pathways in the unfolded protein response: development from yeast to mammals. J. Biochem., 146: 743-750.

Promlek, T., Ishiwata-Kimata, Y., Shido, M., Sakuramoto, M., Kohno, K., and Kimata, Y. 2011. Membrane aberrancy and unfolded proteins activate the endoplasmic reticulum stress sensor Ire1 in different ways. Mol. Biol. Cell, 22: 3520-3532.

Ron, D. and Walter, P. 2007. Signal integration in the endoplasmic reticulum unfolded protein response. Nat. Rev. Mol. Cell Biol., 8: 519-529.

Serrano, R., Kielland-Brandt, M.C., and Fink, G.R. 1986. Yeast plasma membrane ATPase is essential for growth and has homology with (Na+ + K+), K+- and Ca2+-ATPases. Nature, 319: 689-693.

Sikorski, R.S. and Hieter, P. 1989. A system of shuttle vectors and yeast host strains designed for efficient manipulation of DNA in Saccharomyces cerevisiae. Genetics, 122: 19-27.

Umebayashi, K., Hirata, A., Fukuda, R., Horiuchi, H., Ohta, A., and Takagi, M. 1997. Accumulation of misfolded protein aggregates leads to the formation of russell body-like dilated endoplasmic reticulum in yeast. Yeast, 13: 1009-1020.

(Received for publication, August 5, 2019, accepted, October 10, 2019 and published online, October 17, 2019) 